\title{
Severe Kidney Damage in Diabetics on Alternative Medicine
}

\author{
Dalip Ragoobirsing $h^{1 *}$, Errol Morrison ${ }^{2}$ and Herbert Thompson ${ }^{3}$ \\ ${ }^{1}$ Director, Diabetes Education Programme, Faculty of Medical Sciences Teaching \& Research Complex, \\ Level 2, University of the West Indies, Mona Campus, Kingston, Jamaica \\ ${ }^{2}$ Director General and Advisor to the Prime Minister, National Commission on Science and Technology, \\ Kingston, Jamaica
}

${ }^{3}$ Former President, Northern Caribbean University, Mandeville, Manchester, Jamaica

*Corresponding author: Professor Dalip Ragoobirsingh, Director, Diabetes Education Programme, Faculty of Medical Sciences Teaching \& Research Complex, Level 2, University of the West Indies, Mona Campus, Kingston, Jamaica, E-mail: dalip.ragoobirsingh@uwimona.edu.jm

\begin{abstract}
Aim: This study was designed to ascertain whether plant extracts had deleterious effects on patients using it to replace or compliment their prescribed medication. Animal model studies were also conducted to confirm or negate the findings in the patient study.

Methods: Thirteen diabetic patients who had a history of exposure to herbal treatment and/or food toxins were compared to 10 insulin dependent diabetes mellitus (IDDM) patients and 10 normal controls. Each group was matched for age, gender, body mass index (BMI) and in the case of the diabetic controls duration of diabetes. Microalbumin, urinary amylase, glycosylated hemoglobin (HbA1c) and blood pressure were measured using standard techniques. Purified extract from the seeds of the annatto, Bixa Orellana, was fed to anesthetized mongrel dogs $(n=8-14)$. Subsequently, oral glucose tolerance tests and insulin measurements were performed using the standard methods. Concomitant electron microscopy of tissue biopsies was done.
\end{abstract}

Results: Microalbuminuria was significantly higher $(\mathrm{P}<$ 0.05 ) in those that used bush medicines than in the control groups. Urinary amylase concentration was also higher in the former. Confounding factors such as high blood pressure and urinary tract infections were excluded as causes for the differences.

The purified extract demonstrated hyperglycemia in the dog model. Electron microscopy of tissue biopsies revealed damage to major organs especially kidneys and pancreas.

Conclusion: It is apparent that patients who substitute or take bush treatment along with or instead of their prescribed medication suffer severe kidney damage as confirmed by the animal studies.

\section{Keywords}

Alternative medicine, Caribbean, Diabetes mellitus, Dog model, Folklore, Jamaica, Plant extracts

\section{Introduction}

Jamaica is an island in the Caribbean with a population of 2.7 million [1]. As other Caribbean territories, Jamaica has a rich cultural tradition involving the use of herbal remedies. These folklore practices are linked to the immigrants from West Africa and the East Indies as well as the original native Amerindians.

Anecdotal evidence has revealed that people who use folk medicines to treat their diabetes often report that they have been cured because of the low sugar readings obtained on testing. However, these findings could result from masking by components contained in the plant extracts.

Patients have been presenting at the University Hospital of the West Indies (UHWI) with very high blood glucose requiring relatively high doses of insulin followed by a non-insulin requiring phase since 1955 [2]. This clinical picture has presented so frequently that Morrison [3], in 1981, described this group of patients as phasic insulin dependent diabetes mellitus (PIDDM).

This study was undertaken to determine if the aforementioned PIDDM patients suffered any organ damage.

The red powdery extract from the seeds of the an-

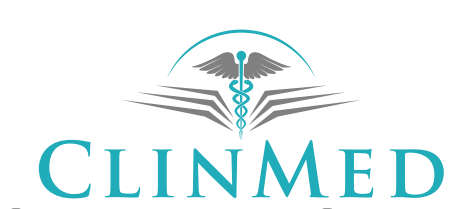

INTERNATIONAL LIBRARY
Citation: Ragoobirsingh D, Morrison E, Thompson H (2018) Severe Kidney Damage in Diabetics on Alternative Medicine. Int J Diabetes Clin Res 5:082. doi.org/10.23937/2377-3634/1410082

Received: September 25, 2017: Accepted: January 20, 2018: Published: January 22, 2018

Copyright: (c) 2018 Ragoobirsingh D, et al. This is an open-access article distributed under the terms of the Creative Commons Attribution License, which permits unrestricted use, distribution, and reproduction in any medium, provided the original author and source are credited. 
natto, Bixa Orellana, is a well-known food coloring. In an oil suspension, it is used as a folk remedy (bush tea) in the West Indies, for diabetes mellitus. Therefore, animal studies, using the annatto extract, were also designed to confirm or negate the findings of the patient study.

\section{Materials and Methods}

Thirteen PIDDM patients attending the Diabetes Outpatient Clinic at the UHWI were studied. These had BMI of $24.7 \pm 0.9 \mathrm{~kg} / \mathrm{m}^{2}$, mean age of $57.0 \pm 3.2$ years and duration of diabetes mellitus of $16.0 \pm 1.3$ years. They were compared with 10 IDDM patients, BMI $23.4 \pm$ $1.9 \mathrm{~kg} / \mathrm{m}^{2}$, mean age $54.0 \pm 5.4$ years, duration of diabetes $14.7 \pm 2.7$ years. The 10 normal controls had BMI of $22.7 \mathrm{~kg} / \mathrm{m}^{2}$ and mean age of $47.0 \pm 4.5$ years. The groups were also matched for gender distribution. Consent was given by all participants of the study.

Microalbuminuria and urinary amylase were measured on $24 \mathrm{~h}$ urine samples using a modification of the method of Silver, et al. [4] and that of Somogyi respectively [5]. Midstream urine samples were investigated for evidence of urinary tract infection. Blood pressure measurements, using standard techniques, were also done on each subject since it is known to affect microalbuminuria. Glycosylated haemoglobin (HbA1c) levels were determined using affinity gel chromatography as described by Gonen, et al. [6].

One-way analysis of variance was used in the human studies. In cases where the value for a particular parameter was skewed a transformation was done. Group means were calculated and post-analysis of variance comparison using Student's t-test was done, as recommended by Armitage [7], in cases where there may have been differences between the diabetic group as a whole and the normal population, provided that there were no intra-diabetic group differences.

Detailed chemical analysis and investigation of the annatto extract (Bixa Orellana) yielded a methyl ester, trans-bixin, molecular weight 394, and molecular formula $\mathrm{C}_{24} \mathrm{H}_{30} \mathrm{O}_{4}$. This is used in the Yucatan and Central America to relieve measles, stomach ache and asthma. In the Caribbean, however, a brew containing trans-bixin is used in the treatment of diabetes mellitus.

This purified substance was fed orally to mongrel dogs $(n=8-14)$. Dogs for this study were secured from The University of the West Indies (UWI) animal house. These were approximately a year old weighing between 12-14 kg each. Dogs were disinfected, washed and fasted for 24 hours before each test. Each dog was weighed and then anesthetized, using sodium pentobarbital 30 $\mathrm{mg} / \mathrm{kg}$ body weight. An endotracheal tube was inserted and then connected to a Palmers Pump to ensure adequate ventilation. An intragastric tube was then inserted for feeding of the appropriate substances. Controls were fed $40 \%$ ethanol, since this was the solvent used in the test animals, followed an hour later with a glucose load, $1.75 \mathrm{~g} / \mathrm{kg}$ body weight. Experimental animals were fed trans-bixin $0.6 \mathrm{mg} / \mathrm{kg}$ body weight dissolved in $40 \%$ ethanol followed an hour later by glucose solution, 1.75 $\mathrm{g} / \mathrm{kg}$ body weight.

A fasting blood sample was taken, by venipuncture, for glucose and insulin determination, using the standard methods. This was followed by the taking of samples for the next three hours.

The study was expanded to feeding the dogs $0.6 \mathrm{~g} / \mathrm{kg}$ body weight of trans-bixin once per month for the next 6 months. GTTs were done 1 hour after the final feeding of trans-bixin.

Electron microscopy of surgically removed tissue biopsies was done. Each dog for surgery was disinfected and washed once per day for three consecutive days prior to surgery. The animal was weighed, shaved and anesthetized using $30 \mathrm{mg} / \mathrm{kg}$ sodium pentobarbital. Adequate ventilation was maintained throughout surgery by way of an inserted endotracheal tube connected to a Palmer Pump respirator. Kidney, pancreas and other samples were taken before $\left(T_{0}\right)$ and after different treatment regimen with the extract for varying periods from 1 to several months. These procedures were all performed in a sterile operating theatre by appropriately trained staff in attendance. Meticulous post-operative care was given to restore the animals to robust health thereafter.

\section{Results}

There were no statistically significant differences in age, BMI and gender distribution between the PIDDM and IDDM patients or between them and the normal controls. The duration of diabetes and $\mathrm{HbA1c}$ did not differ significantly among the groups of patients studied but the latter were increased $(p<0.05)$ in patients when compared to normal controls.

The urine microalbuminuria concentration was significantly higher in the PIDDM group (mean \pm S.E.: 110.5 $\pm 37.6 ; p<0.05)$ than in the IDDM group (38.6 \pm 15.8 $\mathrm{mg} / \mathrm{dl})$. The normal controls had a concentration $16.6 \pm$ $2.3 \mathrm{mg} / \mathrm{dl}$. The urinary amylase concentration per twenty-four hours showed that the IDDM with a concentration of $358.9 \pm 79.7 \mathrm{IU} / 24 \mathrm{~h}$. was well within normal range ( $<570 \mathrm{IU} / 24 \mathrm{~h}$ ). However, the PIDDM group, 902 \pm 242 .0, was significantly higher $(p<0.05)$. Although the volume of urine excreted in a $24 \mathrm{~h}$ period varied, the difference in output between the groups were not statistically significant $(p>0.05)$. The subjects in this study did not show any evidence of urinary tract infections.

Analysis of variance of mean systolic and diastolic pressures of all diabetic patients showed that there was no group difference in blood pressure. Corresponding measurements for the normal controls showed no statistically significant differences between the normal controls and the diabetic groups $(p>0.05)$. 


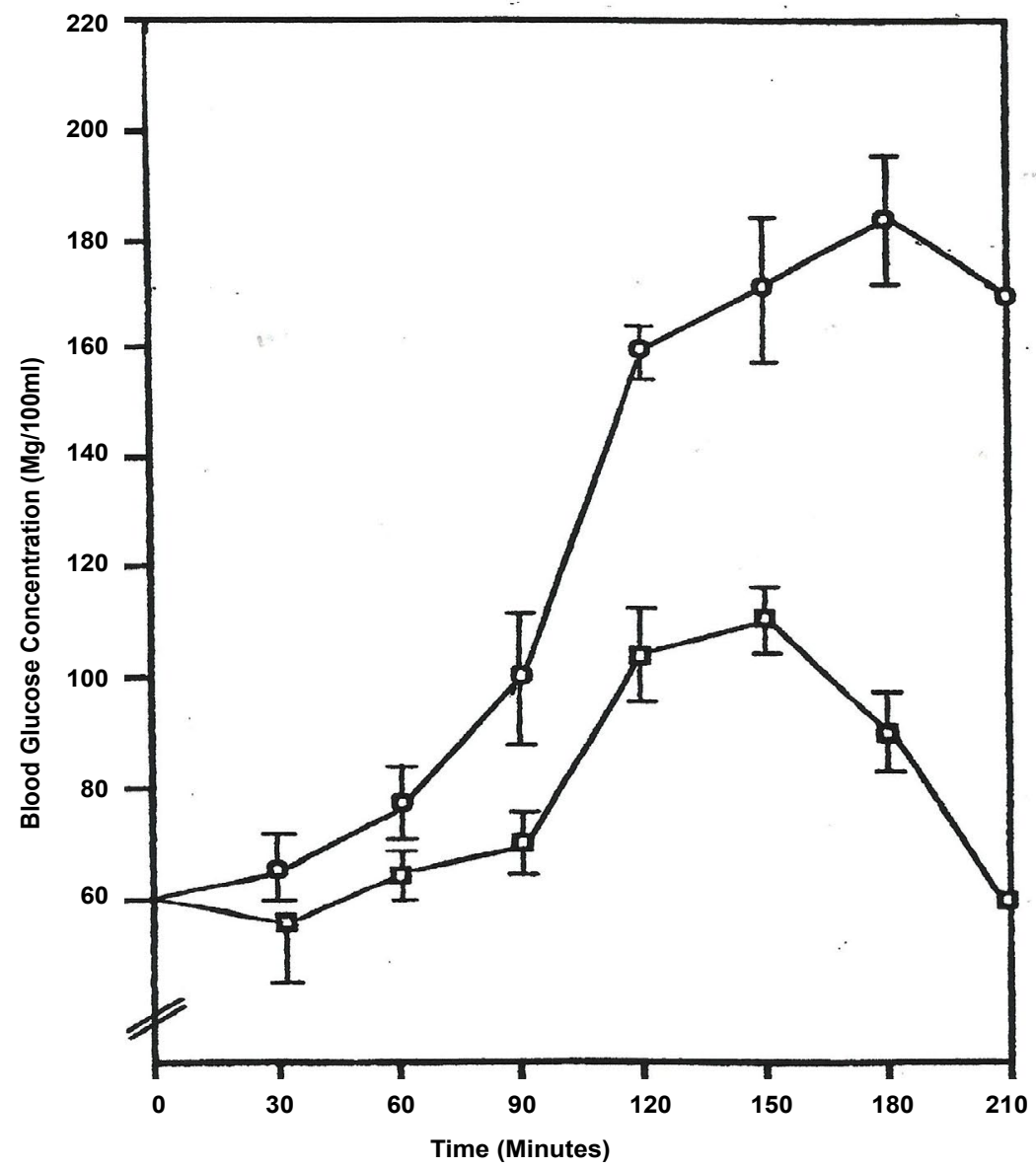

Figure 1: Glucose Tolerance Curve of Dogs Maintained on Trans-Bixin for Six Months.

Values \pm S.E. $M ; \mathrm{N}=8$;

Trans-bixin ( $\mathbf{O}-\mathbf{O})$;

Ethanol control ( $\square-\square)$.

In the animal model studies, the anaesthetized mongrel dogs after repeated treatment with the purified annatto (Bixa Orellana) extract became hyperglycemic (Figure 1). The latter shows a distinctive difference between experimental and control groups beginning at the 90 minutes interval. This gap widens at each subsequent interval. Matched t-test analyses showed an appreciable difference between the two groups $(p<0.05)$ and a highly significant difference between the 120 and 180 intervals $(p<0.01)$.

Figure 2 shows the mean results \pm SEM of insulin levels in each dog $(n=14)$ for each time interval. In matched t-test between the control and single fed dogs the variation in insulin levels were highly significant ( $p$ $<0.01$ ). A similar statistical analysis done on the multiple-fed and the control showed significant differences $(p<0.03)$. When the two experimental group were analyzed, using unmatched t-test, the difference in insulin levels were significant only beyond the 60 minute marker $(p<0.05)$. Of note, the figure also shows that insulin levels continue to rise in both experimental groups beyond the 150 minutes marker, when ordinarily there is a decline in concentrations at this point and beyond.

Electron photomicrograph of kidney tissue from ethanol control dogs show healthy convoluted tubular cells with large nuclei, ducts with intact basement membranes along its walls, glomeruli with its various capillaries and large intact mitochondria.

On the other hand, electron photomicrographs of kidney tissue from dogs multiple- fed with trans-bixin within a month show destruction of mitochondria in the distal convoluted tubular cells, still other areas show 'empty cases' indicative of complete wasting of mitochondria and other organelles in the cytoplasm. Widespread necrosis and accumulation of cell debris in the brush border areas were also noted.

Microscopic examination of pancreatic acinar cells from control showed intact zymogen granules, intact mitochondria, large nuclei and a proliferation of endoplasmic reticulum throughout.

Within a month of feeding trans-bixin, electron photomicrographs show destruction of zymogen granules in the acinar area. Beta cells were seen with granules bearing insulin rods damaged and intact. Mitochondrial damage was also evident. Increased exposure to trans-bixin resulted in a progressive pattern of destruction to various organelles with a central accumulation of darkened zymogen debris. A breakdown of internal structures of organelles in a manner more disruptive 


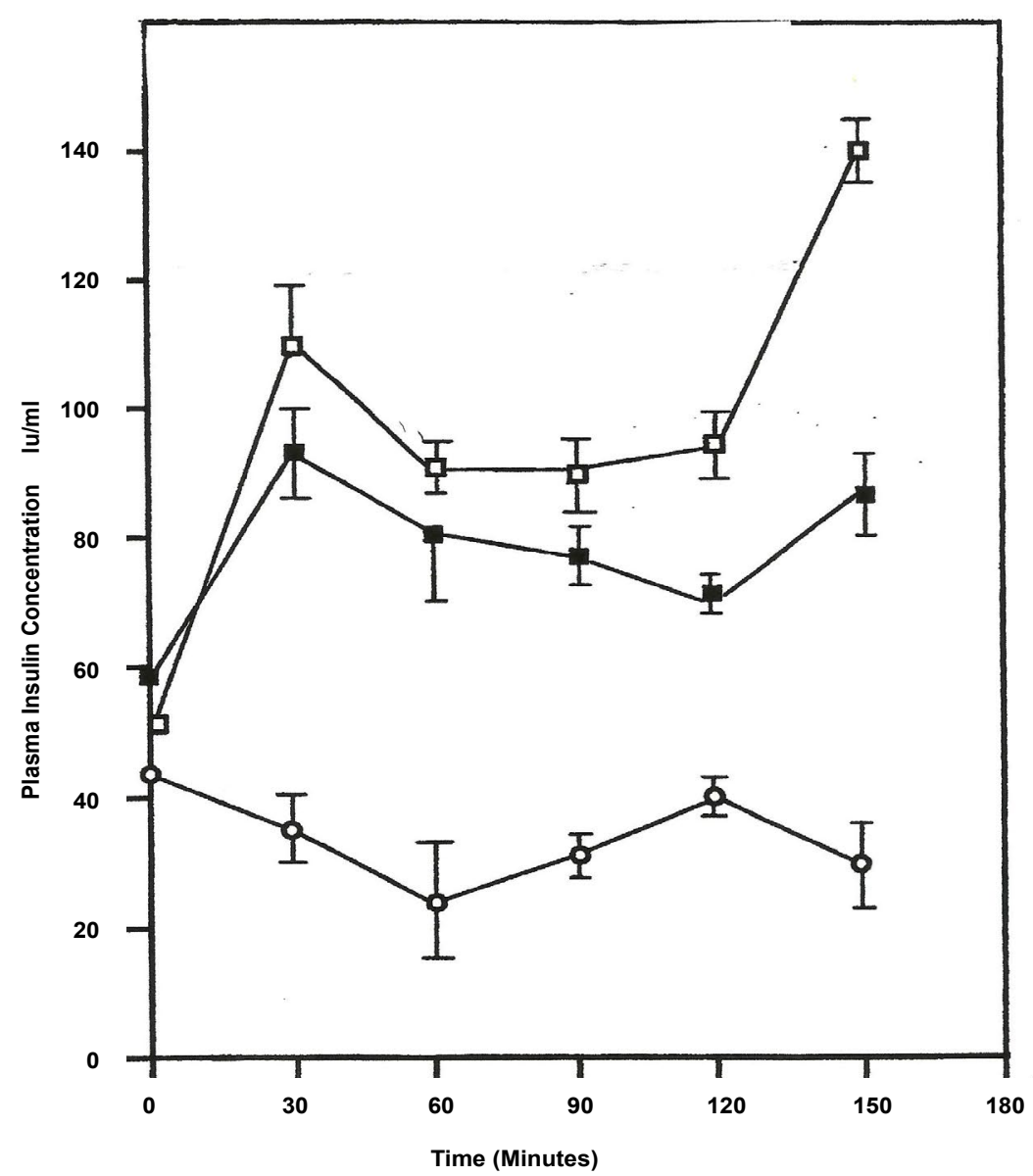

Figure 2: Effects of Trans-Bixin on Plasma Insulin Levels.

Values \pm S.E. $M$; $N=14$;

Single feeding of trans-bixin ( $\square-\square)$;

Repeated feeding of trans-bixin ( $\square-(\square)$ );

Ethanol control $(\mathbf{O}-\mathbf{O})$.

and widespread than stacking of cristae evident in other tissues exhibiting damage.

\section{Discussion}

Severe kidney damage in the PIDDM patients was intimated by the high urinary amylase discharged. Similar to other low molecular weight proteins filtered by glomeruli, amylases are reabsorbed to an extent by the proximal tubule. Thus, conditions associated with increased production and glomerular filtration of other low molecular weight proteins that compete with tubular reabsorption of amylase, or conditions of proximal tubular injury may increase urinary amylase excretion [8]. This finding, in this group of patients, suggests a renal defect in either the nephron structure and/or function. This was confirmed by the astronomical microalbuminuria seen in this group. The IDDM excreted microalbumin in quantities which exceeded that of the normal $(p<0.05)$ which is indicative of the diabetic nephropathic changes anticipated. However, the exceedingly high output seen in the PIDDM which was approximately three times that seen in the IDDM suggests that the toxic substances in the herbal remedies used by the former could be responsible.
This becomes clearer given that the diabetic groups were matched for duration of diabetes. Also, the level of control in both groups was similar as evidenced by the comparable glycosylated hemoglobin. It is a well-established clinical fact that the $\mathrm{HbA1c}$ index has become the gold standard for long-term monitoring of glycemic control and is a validated measurement tool for assessing diabetes status [9].

The fact that no pus cells or bacterial overgrowth was detected in the mid-stream urine samples of the diabetic groups was another corroborative finding. This is very important in interpreting microalbumin results, since the presence of an occult infection can lead to spurious interpretation. This is particularly important in the diabetic since they have a higher incidence of urinary tract infections than do non-diabetics. Predisposition to urinary tract infections in diabetes mellitus results from several factors. Susceptibility increases with longer duration and greater severity of diabetes. High urine glucose content and defective host immune factors predispose to infection [10].

Another confounding factor was hypertension. Normal glomeruli do not allow protein leakage from the 
capillary lumen into the urine. However, a significant increase in intracapillary pressure or structural damage of the glomerular membrane may lead to the leakage of protein from the plasma into Bowman's space, which contains the initial urinary fluid. Thus, the process leading to the development of microalbuminuria may vary with the severity of hypertension. A hemodynamic cause related to significant increases in intraglomerular capillary pressure may be more likely in mild hypertension, whereas glomerular injury may be more significant in moderate-to-severe hypertension [11]. However, increased blood pressure could not be the cause of the excessive microalbuminuria seen in the PIDDM since there was no significant difference in systolic and/or diastolic pressure between the diabetic groups.

The findings of the animal studies lend credence to the aforementioned hypothesis. The methyl ester, trans-bixin, is a potential free radical which is a source of lipid peroxidation. The latter is a free radical process involving a source of secondary free radical, which further can act as a second messenger or can directly react with other biomolecules, enhancing biochemical lesions. Lipid peroxidation occurs on polyunsaturated fatty acid located on the cell membranes which can account for the cellular damage seen in the electron micrographs [12]. This may account for the pancreatic insult resulting in the decreased plasma insulin and resultant hyperglycemia in the treated animals.

The trans-bixin molecule also lends itself readily to formation of a hydroxyl radical which is thought to initiate reactive oxygen species (ROS) [13] and remove hydrogen atom, thus producing a lipid radical which is further converted into a diene conjugate. Further, by addition of oxygen it forms a peroxyl radical; this highly reactive radical attacks another fatty acid forming lipid hydroperoxide and a new radical. Thus lipid peroxidation is propagated. Due to lipid peroxidation, a number of compounds are formed, for example, alkanes, malondialdehyde and isoprostanes. These compounds are used as markers in lipid peroxidation assay and have been verified in many diseases including diabetes [14]. This cascade of events could be responsible for the severe damage [15] noted in the electron micrographs of the kidneys after 1 month of treatment as well as the pancreas. Prolonged exposure to free radicals, even at low concentration, may result in the damage of biologically important molecules and potentially lead to DNA mutation, tissue injury and disease [16].

At the sub-cellular level, trans-bixin caused widespread mitochondrial damage to the kidney and the pancreas. There was mitochondrial stacking, swelling and rupture. Trans-bixin is known to disrupt events in the respiratory chain resulting in lower adenosine triphosphate (ATP) production. Water taken into the mitochondria by osmosis is normally removed by the action of ATP possibly through an actomyosin-like contraction serving as a pump [17]. In the aforementioned absence of ATP the water will necessarily accumulate and cause swelling of the mitochondrion. This could explain, if only in part, the large-scale mitochondrial swelling and rupture seen especially in the kidneys.

Considering the extent of pancreatic damage effected it seems reasonable to conclude that trans-bixin acts by a similar mechanism, as does the diabetogenic agents alloxan and streptozotocin. These toxic glucose analogues preferentially accumulate in pancreatic beta cells via the GLUT2 glucose transporter. As suggested above of trans-bixin, alloxan generates ROS in a cyclic redox reaction with its reduction product, dialuric acid. Autoxidation of dialuric acid generates superoxide radicals, hydrogen peroxide and, in a final iron-catalyzed reaction step, hydroxyl radicals. These hydroxyl radicals are ultimately responsible for the death of the beta cells, which have a particularly low antioxidative defense capacity, and the ensuing state of insulin-dependent 'alloxan diabetes'. As a thiol reagent, alloxan also selectively inhibits glucose-induced insulin secretion through its ability to inhibit the beta cell glucose sensor glucokinase. Following its uptake into the beta cells, streptozotocin is split into its glucose and methylnitrosourea moiety. Owing to its alkylating properties, the latter modifies biological macromolecules, fragments DNA and destroys the beta cells, causing a state of insulin-dependent diabetes. The targeting of mitochondrial DNA, thereby impairing the signaling function of beta cell mitochondrial metabolism, also explains how streptozotocin is able to inhibit glucose-induced insulin secretion [18].

These studies suggest, if not confirm, that patients who use alternative medicine along with or instead of their prescribed medication are at greater risk for severe diabetic nephropathy while exacerbating their diabetic condition than those who comply. The more severe renal dysfunction is an adverse prognostic factor for the survival of these patients.

As such the authors use this medium to, respectfully, suggest where scientific support is not forthcoming to explain any benefit of the use of a medicinal plant extract, then it should be discouraged and cautioned against as a possible harmful practice.

\section{Acknowledgement}

The authors recognize the monumental contributions of colleagues, especially Drs. K. Pascoe (chemist), C. Fletcher (surgeon), Professor M. West (pharmacologist), Nurse E. West, Staff of the Pathology departments and animal house, UWI and Loma Linda University, USA. Funding was provided by the UWI, Mona Campus, Jamaica.

\section{Conflict of Interest}

The authors state categorically that we have no fi- 
nancial or personal relationship with other people or organizations that could inappropriately influence the work therein contained.

\section{Ethical Statement}

The protocols for the research reported in this paper conducted on humans and animals were reviewed and approved by the appropriate Ethics Committee of the University of the West Indies. They were satisfied that the procedures were appropriate and humane.

\section{References}

1. Demographic Statistics (2005) Statistical Institute of Jamaica.

2. Hugh-Jones $P$ (1955) Diabetes in Jamaica. Lancet 891 895.

3. Morrison EY (1981) Diabetes Mellitus: A third syndrome (Phasic Insulin Dependence). International Diabetes Federation Bulletin 26: 784-787.

4. Clark P, McDonald T (2013) Diabetes Mellitus. In: Wild D, The Immunoassay Handbook: Theory and application of ligand binding. Elsevier, Massachusetts, USA, 783-794.

5. Teitz WW (1987) Fundamentals of clinical chemistry. WB Saunders, Philadelphia, USA, 1-1010.

6. Gerber SM, Stickle DF, Ahmed I, Jabbour SA (2015) Glycated hemoglobin serum proteins and other markers as tools for monitoring. In: DeFronzo RA, Ferrannini E, Alberti G, Zimmet P, International Textbook of Diabetes Mellitus. John Wiley \& Sons, Sussex, UK, 853-868.

7. Armitage $P(1980)$ Statistical Methods in Medical Research. In: Armitage D, Great Britain Blackwell Scientific Publications, 382-392.
8. Treacy J, Williams A, Bais R, Willson K, Worthley C, et al. (2001) Evaluation of amylase and lipase in the diagnosis of acute pancreatitis. ANZ Journal of Surgery 71: 577-582.

9. American Association of Clinical Endocrinologists (2000) The American Association of Clinical Endocrinologists Medical Guidelines for the Management of Diabetes Mellitus: The AACE system of intensive diabetes self-management -2002 update. Endocr Pract 6: 43-84.

10. Chen SL, Jackson SL, Boyko EJ (2009) Diabetes mellitus and urinary tract infection: Epidemiology, pathogenesis and proposed studies in animal models. J Urol 182: S51-S56.

11. Karalliedde J, Viberti G (2004) Microalbuminuria and Cardiovascular Risk. American Journal of Hypertension 17: 986-993.

12. Kumar V, Abbas AK, Fausto N (2009) Cellular Adaptations, cell injury and cell death. In: Robbins and Cotran, Pathologic basis of disease. Saunders, Philadelphia, USA, 15-18.

13. Sen S, Chakraborty R, Sridhar C, Reddy YSR, De B (2010) Free radicals, antioxidants, diseases and phytomedicines: Status and future prospect. International Journal of Pharmaceutical Sciences Review and Research 3: 91-100.

14. Lobo V, Patil A, Phatak A, Chandra N (2010) Free radicals, antioxidants and functional foods: Impact on human health. Pharmacogn Rev 4: 118-126.

15. Rao S, Kalva S, Yerramilli A, Mamidi S (2011) Free radicals and tissue damage: Role of antioxidants. Free Radical and Antioxidants 1: 2-7.

16. Fang YZ, Yang S, Wu G (2002) Free radicals, antioxidants and nutrition. Nutrition 18: 872-879.

17. Stachowiak MR (2011) Mechanisms of actomyosin contractility in cells. Columbia University Academic Commons.

18. Lenzen S (2008) The mechanisms of alloxan- and streptozotocin-induced diabetes. Diabetologia 51: 216-226. 